\title{
HeteroPar'2010: Eighth International Workshop on Algorithms, Models and Tools for Parallel Computing on Heterogeneous Platforms
}

\author{
Frédéric Vivien \\ LIP, École normale supérieure de Lyon, and INRIA, France
}

\section{Foreword}

Networks of computers are now the most common and available parallel architecture. Unlike dedicated parallel computer systems, networks are inherently heterogeneous. They consist of diverse computers of different performance interconnected via heterogeneous network equipment providing communication links with different latencies and bandwidths. Traditional parallel algorithms and tools are aimed at homogeneous multiprocessors and cannot be efficiently used for parallel computing on heterogeneous networks. New ideas, dedicated algorithms and tools are needed to efficiently use this new type of parallel architecture.

The HeteroPar workshop series is intended to be a forum for people working on algorithms, programming languages, tools, and theoretical models aimed at efficient problem solutions on heterogeneous networks. The covered topics target heterogeneous systems and platforms, and include parallel programming languages and libraries, fault tolerance, tools for grid, cloud and green computing, and the usage of these complex platforms for solving different types of problems and applications.

HeteroPar'2010 was the eighth edition of this workshop, and the second one co-located with the Euro-Par conference. Out of 12 manuscripts submitted this year, 7 were accepted for presentation at the Workshop in Ischia on August 30 . Each submission received 4 reviews. Apart from the presentation of the 7 accepted papers, the workshop had one invited speaker of international reputation, Marco Danelutto, who talked about Structured programming models targeting heterogeneous architectures.

As program chair, I wish to acknowledge all those who contributed to the success of HeteroPar'2010, in particular to the authors of the submitted papers, to the Program Committee members for their invaluable time and expertise, and to the organizers of EuroPar 2010. 